\title{
User's Guide for mapIMG 3-Map Image Re-Projection Software Package
}

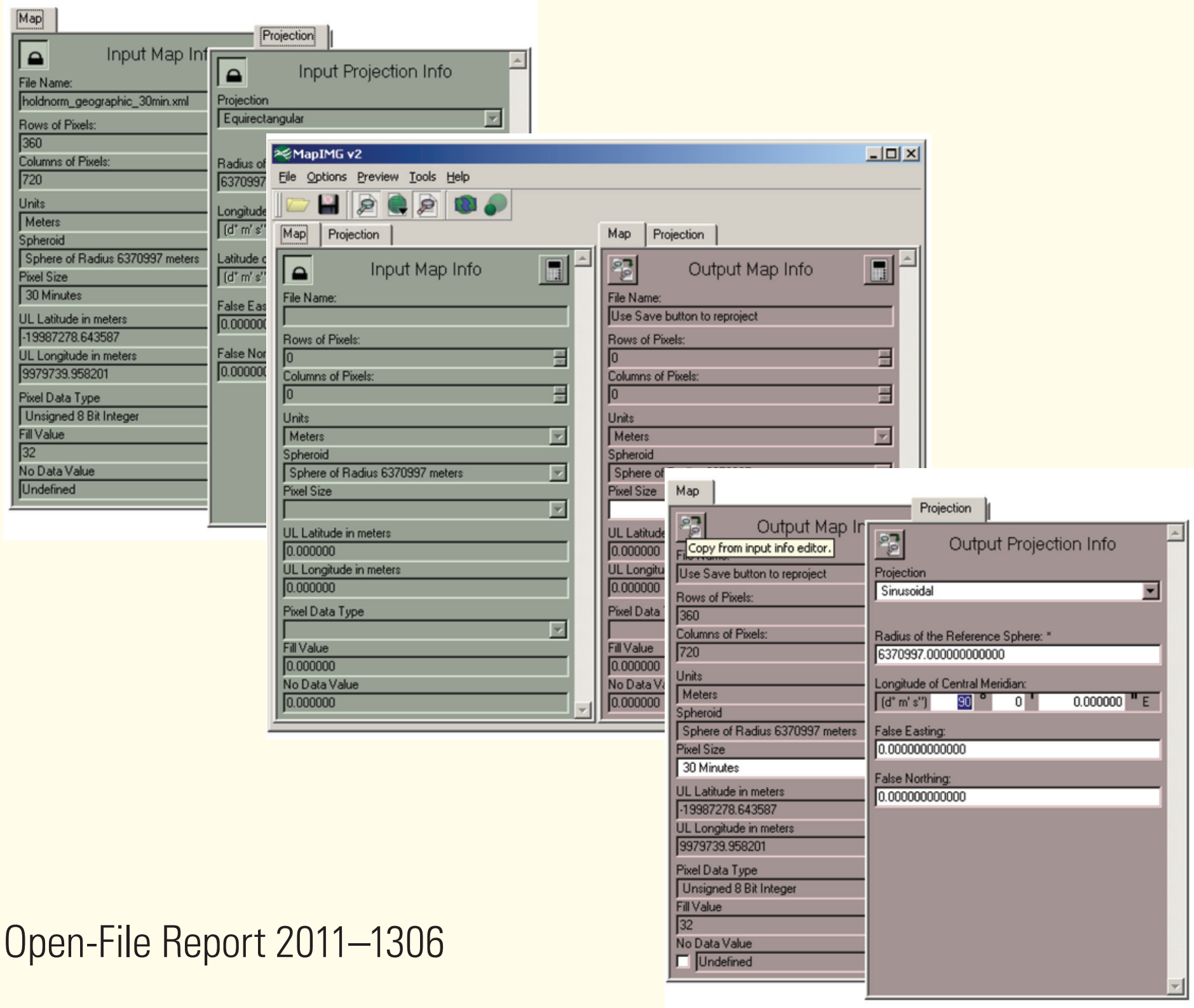


Cover. Screen captures from the software program. 


\section{User's Guide for mapIMG 3-Map Image Re-Projection Software Package}

By Michael P. Finn and David M. Mattli

Open-File Report 2011-1306

U.S. Department of the Interior

U.S. Geological Survey 


\title{
U.S. Department of the Interior \\ KEN SALAZAR, Secretary \\ U.S. Geological Survey \\ Marcia K. McNutt, Director
}

\author{
U.S. Geological Survey, Reston, Virginia: 2012
}

For more information on the USGS - the Federal source for science about the Earth, its natural and living resources, natural hazards, and the environment, visit http://www.usgs.gov or call 1-888-ASK-USGS.

For an overview of USGS information products, including maps, imagery, and publications, visit http://www.usgs.gov/pubprod

To order this and other USGS information products, visit http://store.usgs.gov

Any use of trade, product, or firm names is for descriptive purposes only and does not imply endorsement by the U.S. Government.

Although this report is in the public domain, permission must be secured from the individual copyright owners to reproduce any copyrighted materials contained within this report.

Suggested citation:

Finn, M.P., and Mattli, D.M., 2012, User's guide for mapIMG 3-Map image re-projection software package: U.S. Geological Survey Open-File Report 2011-1306, 12 p. 


\section{Contents}

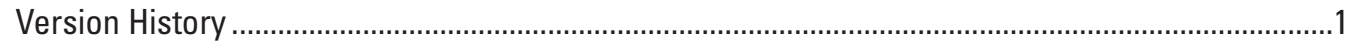

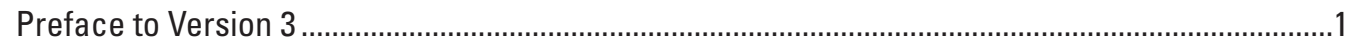

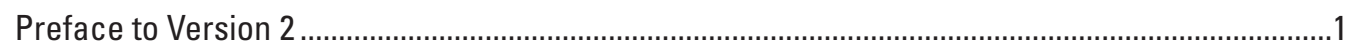

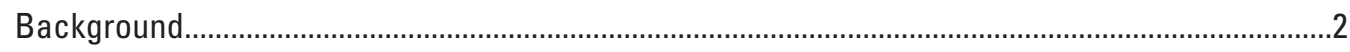

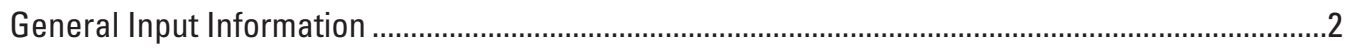

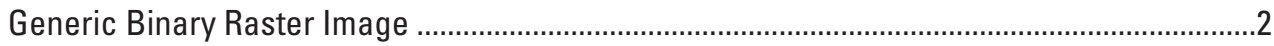

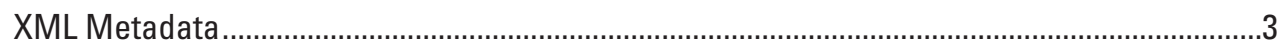

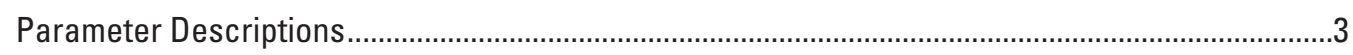

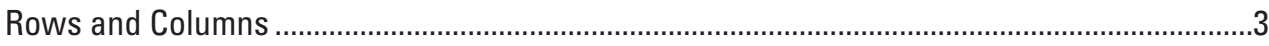

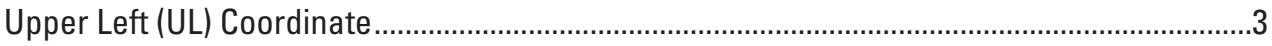

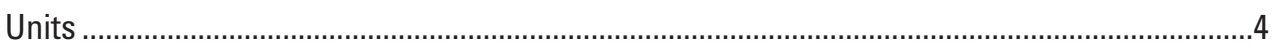

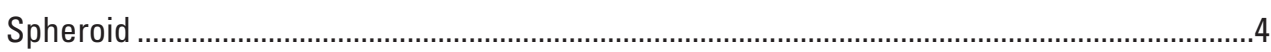

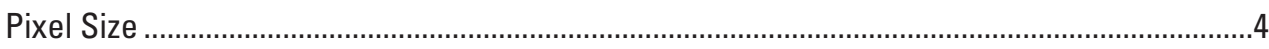

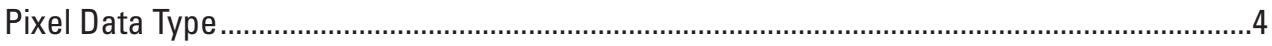

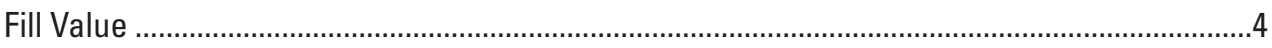

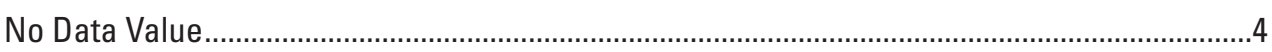

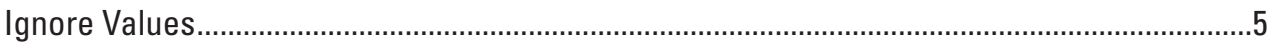

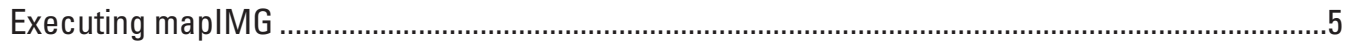

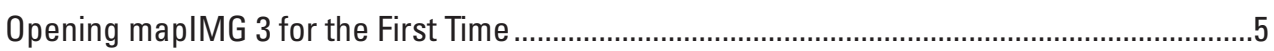

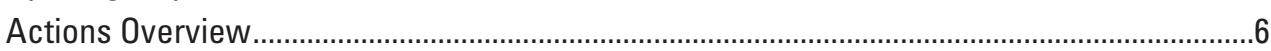

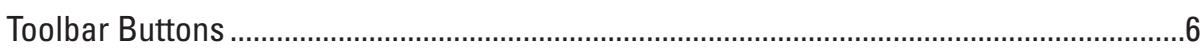

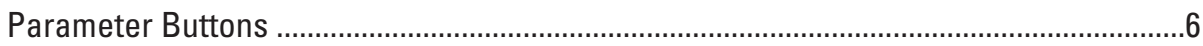

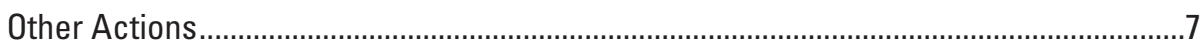

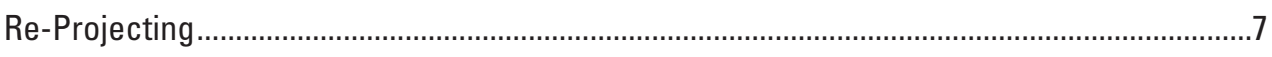

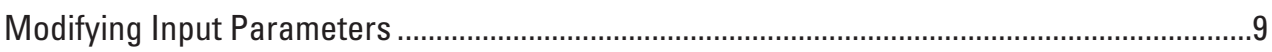

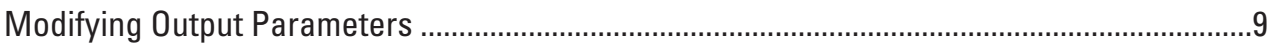

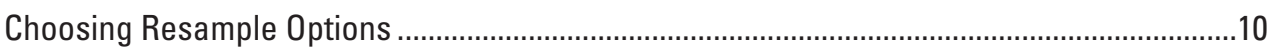

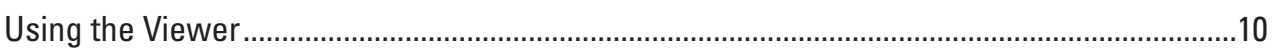

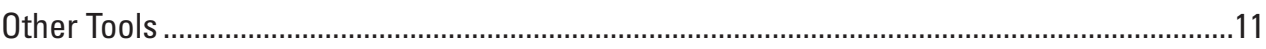

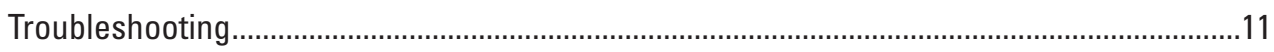

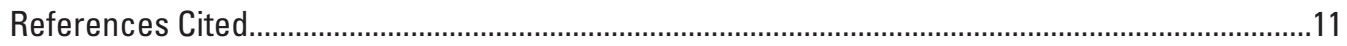




\section{Figures}

1. Screen capture showing Bad Projection tag..................................................................

2. Screen capture showing Bad Projection Parameters Error Message ..............................2

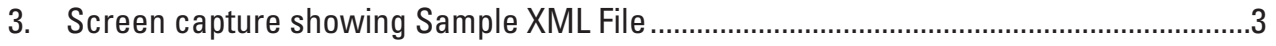

4. Screen capture showing Cell Reference.........................................................................

5. Screen capture showing Author Properties Dialog.......................................................5

6. Screen capture showing mapIMG Main Dialog ……................................................5

7. Screen capture showing Open File Dialog ..................................................................

8. Screen capture showing Loaded Input Parameters ............................................................

9. Screen capture showing Edited Output Parameters..........................................................

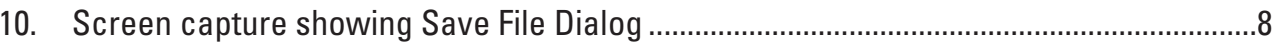

11. Screen capture showing Resample Options Dialog ........................................................

12. Screen capture showing Re-projection Progress............................................................

13. Screen capture showing Completed Message Box ……................................................

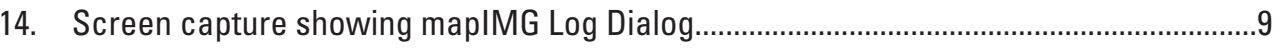

15. Screen capture showing Missing or Corrupt XML ......................................................

16. Screen capture showing Ignore Values Section ..............................................................11 


\title{
User's Guide for mapIMG 3-Map Image Re-Projection Software Package
}

\author{
By Michael P. Finn and David M. Mattli
}

\section{Version History}

Version 0.0 (1995), Dan Steinwand, U.S. Geological Survey (USGS)/Earth Resources Observation Systems (EROS) Data Center (EDC) - Version 0.0 was a command line version for UNIX that required four arguments: the input metadata, the output metadata, the input data file, and the output destination path.

Version 1.0 (2003), Stephen Posch and Michael P. Finn, USGS/Mid-Continent Mapping Center (MCMC_-Version 1.0 added a GUI interface that was built using the Qt library for cross platform development.

Version 1.01 (2004), Jason Trent and Michael P. Finn, USGS/MCMC - Version 1.01 suggested bounds for the parameters of each projection. Support was added for larger input files, storage of the last used input and output folders, and for TIFF/ GeoTIFF input images.

Version 2.0 (2005), Robert Buehler, Jason Trent, and Michael P. Finn, USGS/National Geospatial Technical Operations Center (NGTOC) - Version 2.0 added Resampling Methods (Mean, Mode, Min, Max, and Sum), updated the GUI design, and added the viewer/pre-viewer. The metadata style was changed to XML and was switched to a new naming convention.

Version 3.0 (2009), David Mattli and Michael P. Finn, USGS/Center of Excellence for Geospatial Information Science (CEGIS)_-Version 3.0 brings optimized resampling methods, an updated GUI, support for less than global datasets, UTM support and the whole codebase was ported to Qt4.

\section{Preface to Version 3}

Major changes for this release include support for the Universal Transverse Mercator (UTM) projection, faster reprojection, input/output (I/O) of less-than-global datasets, and numerous bug fixes. Several projections are still not supported and so the caveat in the preface for Version 2 still applies.

Several large changes have been made to the implementation of the user interface but user-level changes should be minimal. A user of the previous version of mapIMG should be able to immediately use this version, noticing only faster re-projection and new features.

\section{Preface to Version 2}

Major significant changes for version 2.0 include a new user interface to be more similar to standard window applications, a viewer to show loaded files and preview re-projections, different resample methods beyond nearest neighbor, and XML files (a popular ASCII file structure for containing structured information) as the new format for the metadata files. Author, fill value, and no data value information have been added to the metadata file (see 'General Input Information' for additional details). A naming convention also has been selected such that mapIMG is the new spelling; it will no longer be referred to as mapimg.

More projections are supported with mapIMG 2 than were supported in mapIMG v1.01, but a few still remain unsupported. However, the control has now been given to the user to run these special projections at his or her risk. Certain projections are marked with a "Bad Projection" tag as shown in figure 1 .

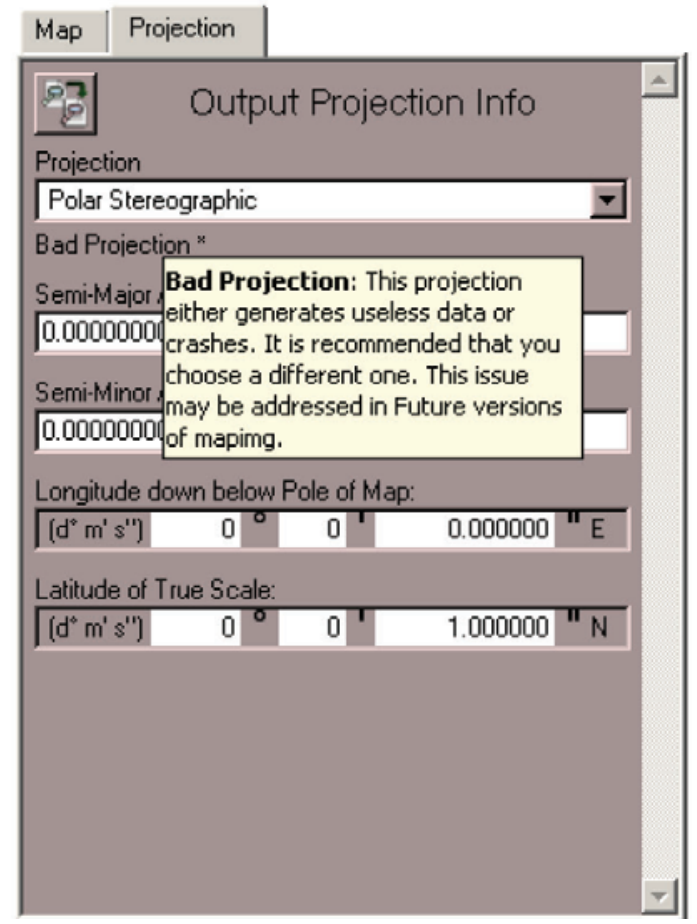

Figure 1. Bad Projection tag. 
If the user attempts to continue with this projection, an error message will pop-up (fig. 2). By clicking "Ignore" the user can continue and mapIMG will attempt to perform the re-projection. More often than not this course of action will result in a map of zero size, large amounts of extraneous data, or a crash.

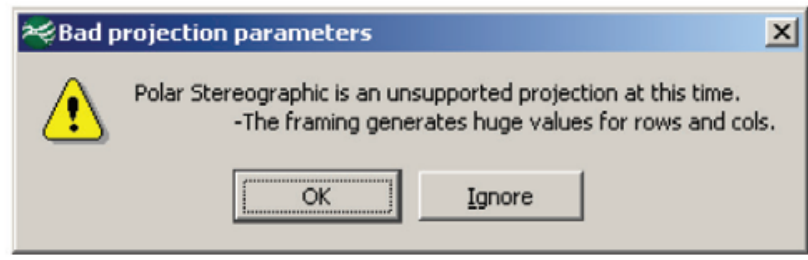

Figure 2. Bad Projection Parameters Error Message.

This feature should be quite useful if the user is a developer/programmer who is attempting to tweak a "Bad Projection." An example would be working with the Polar Stereographic projection. The mapIMG 2 program transforms points near the opposite pole to be at or near infinite. If the developer reprograms mapIMG to skip points within 10 degrees of the opposite side, then running the projection would require choosing "Ignore" as opposed to finding and altering the code that labels Polar Stereographic as a "Bad Projection."

It should be noted that, mapIMG 2 only supports raster datasets of global extent; therefore, calculated values only will be valid if the input is truly to a global extent.

Although this user's guide does make reference to previous versions, it also is complete for the current version of mapIMG. All features of the mapIMG 2 software package are explained in this document.

\section{Background}

Scientists routinely accomplish small-scale geospatial modeling in the raster domain, using high-resolution datasets for large parts of continents and low-resolution to high-resolution datasets for the entire globe. Direct implementation of point-to-point transformation with appropriate functions yields the variety of projections available in commercial software packages, but implementation with data other than points requires specific adaptation of the transformation equations or prior preparation of the data to allow the transformation to succeed. It seems that some of these packages use the General Cartographic Transformation Package (GCTP; Elassal, 1987) or similar point transformations without adaptation to the specific characteristics of raster data (Usery and others, 2003a).

Usery and others (2003b) compiled and tabulated the accuracy of categorical areas in projected raster datasets of global extent. Based on the shortcomings identified in these studies, geographers and applications programmers at the U.S. Geological Survey (USGS) expanded and evolved a USGS software package, mapIMG, for raster map projection transformation (Finn and Trent, 2004). Daniel R. Steinwand of the USGS' Earth Resources Observation and Science Center, originally developed mapIMG, basing it on GCTP (Usery and others, 2001). Through previous and continuing efforts at the USGS, this program has been transformed from an application based on command line input into a software package based on a graphical user interface for Windows, Linux, and other Unix (officially trademarked as UNIX) machines.

mapIMG 3 is on the Web. Source code and binaries available for download at: http://carto-research.er.usgs.gov/ projection/acc_proj_data.html.

\section{General Input Information}

\section{Generic Binary Raster Image}

The primary file type of mapIMG is a Generic Binary raster file. mapIMG uses .img as the extension for these files. They contain no header data. The file is composed entirely of values for cells sorted in row major order. Currently, mapIMG can manipulate and view files with any one of a number of data types used to store the values. These data types are unsigned or signed, 8-, 16-, or 32-bit integers, and 32- or 64- bit floats. Single-image tagged image file format (TIFF) or GeoTIFF files also may be used as input; however, it is highly recommended that all images be exported to Generic Binary if at all possible.

If TIFF/GeoTIFF images are used, they are exported to Generic Binary format as soon as they are loaded. The TIFF format stores images as 32-bit color values, which are then extracted to Generic Binary. It is recommended that TIFF input be used only for thematic data; continuous data will lose all actual values and only relative values will remain.

Band interleaved by line (BIL) is a storage format for image data. For a single band of data, BIL is the same as band sequential (BSQ) and band interleaved by pixel (BIP) (Slater, 1980). Generic Binary, as used in mapIMG (consistent with ERDAS Imagine), is any of these single-band formats that store data in a left-to-right, top-to-bottom row order (ERDAS, 1999). It allows for varying data types - for example, from 8 to 64-bit data.

CAUTION.-The Generic Binary file must use the same byte ordering (endian) as the computer running mapIMG. The file is a raw image with absolutely no metadata, not even the dimensions of the image, which must be known by the user. The output also will be in the same raw format.

A common naming convention is recommended to keep track of files: in particular, the format (type)_(proj)_(pix_size).img, 
where type is the type of data (theme) being used, proj is the projection used, and pix_size is the output file's pixel size. For example, glc_sine_30sec.img would be used to represent a global land cover image in the sinusoidal projection with a pixel size of 30 arc-seconds. Notice that the file name always ends in .img, signifying a generic binary image. Although these conventions are not mandatory for proper program execution, they provide a useful and reliable way to keep track of files.

\section{XML Metadata}

Whenever mapIMG is executed on a file, an associated XML file (fig. 3) will be created from the parameters selected by the user for both the input and the output image. For an input/output file called file_name.img, the associated XML file will be named file_name.xml. It is not absolutely necessary to save these files, but it is highly recommended. If the input/output files are to be used again in the future, the XML files will make sure the correct projection parameters are used.

\section{Parameter Descriptions}

\section{Rows and Columns}

Rows and Columns define the pixel dimensions of the generic binary raster image.

\section{Upper Left (UL) Coordinate}

UL Coordinate is the measurement, in meters, that anchors the upper left corner, the "first cell", of the image. The "first" cell is usually located at $90^{\circ} \mathrm{N} 180^{\circ} \mathrm{W}$ (as in this implementation for global map projections). It is measured from the location of the UL coordinate of the "center" cell of the image (raster file) that is generally located at $0^{\circ}$ latitude $0^{\circ}$ longitude, but can be anywhere the mapmaker defines it. The UL latitude measurement is the number of meters from the "center" cell to the "first" cell in the northward direction. Likewise, the UL longitude measurement is the number of meters from the "center" cell to the "first" cell in the westward direction. Figure 4 pictorially depicts the previous statements.

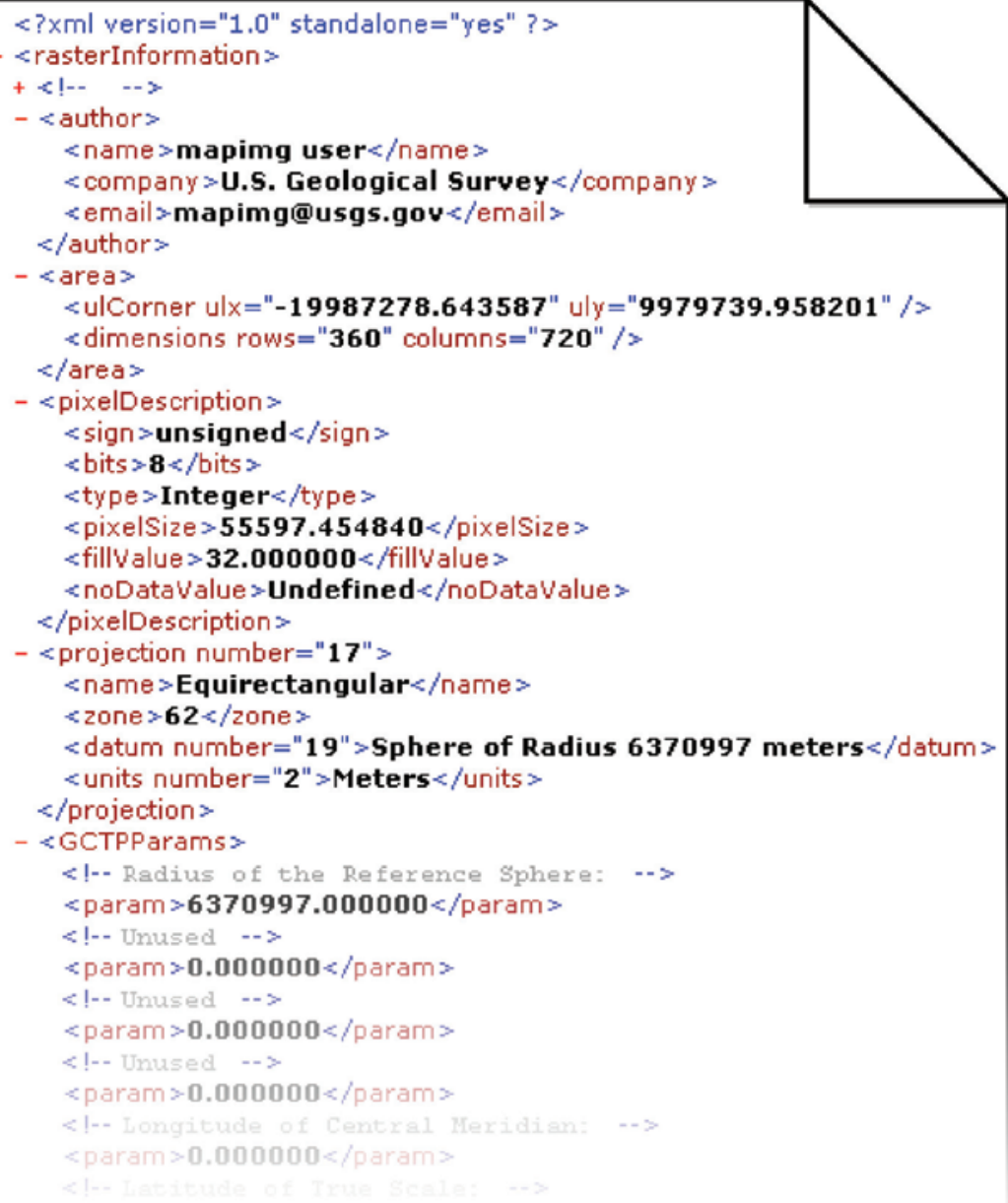

Figure 3. Sample XML File. 


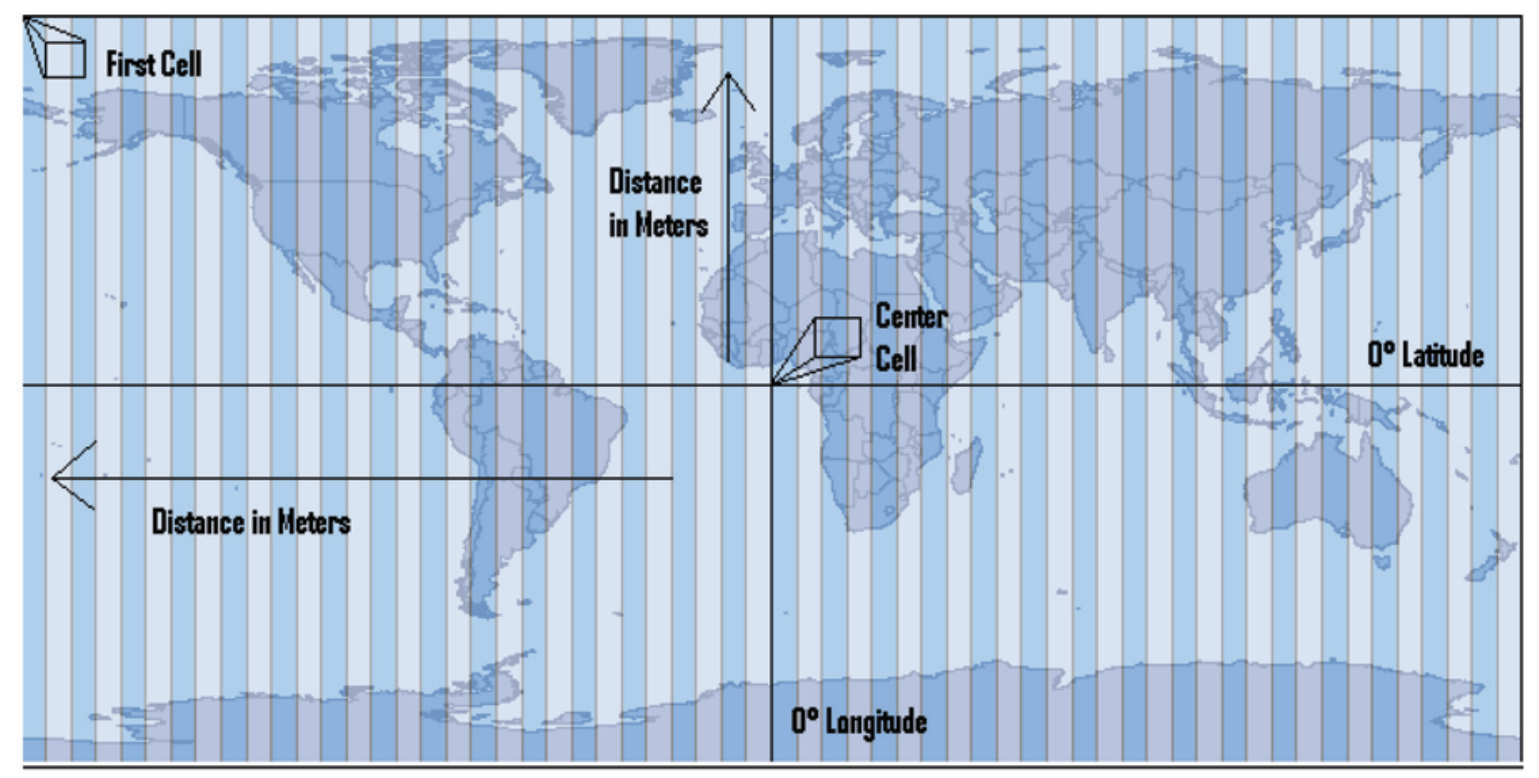

Figure 4. Cell Reference.

\section{Units}

Units define the units of measurement for each of the parameters. Currently only meters are supported. If changing units are supported in the future then it would be possible to enter parameters such as the upper left coordinate in units other than meters.

\section{Spheroid}

Spheroid defines the shape of the earth for use in the projections. This is another parameter that cannot be adjusted at this time. Only a spheroid of radius $6,370,997$ meters currently (2012) is supported.

\section{Pixel Size}

Pixel Size describes the width and height in meters of a pixel at the point of true scale in the image.

\section{Pixel Data Type}

Pixel Data Type describes the way each pixel's value is stored in the image file (for example, 8-Bit Unsigned Integer and 32-Bit Float).

\section{Fill Value}

Fill Value is the numeric value that mapIMG places in areas that are not part of the map.

\section{No Data Value}

No Data Value is used to determine places in a map where no data are present. This value never occurs outside the map where fill value would be placed. It is used by mapIMG for resampling methods where the sample being pulled from the input is full of ignore values. 


\section{Ignore Values}

Ignore Values are entered on the resample form in a list at the same time that the parameters for the output image are changed. All values listed will not be used in calculations during the resample. That is, they are not part of the re-projection, but are there only so there will be no holes between pixels inside or outside the map frame. The no "data value" and "fill value" also are ignored.

\section{Executing mapIMG}

mapIMG is a stand-alone program available for Windows, Linux, and Solaris. All images in this guide are of the Windows version, but other versions will look similar. Double click the mapIMG executable (mapIMG3.exe) to begin execution of the program.

\section{Opening mapIMG 3 for the First Time}

The first time mapIMG 3 is run, the Author Properties dialog will appear as shown in figure 5 .

After entering a name, company, and email address, click "Ok" to save them and continue. Clicking "Cancel" will

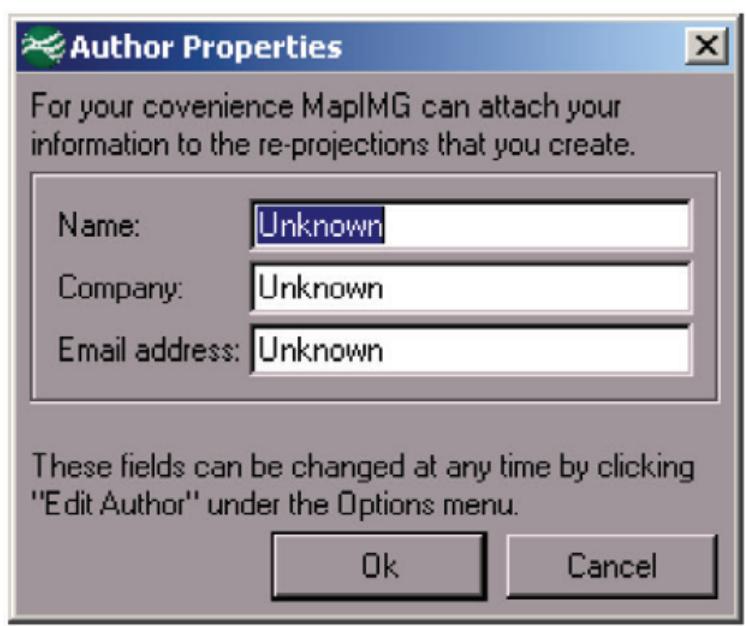

Figure 5. Author Properties Dialog.

continue without saving. The mapIMG main dialog shown in figure 6 will appear, and mapIMG is ready for use.

If at any time the author fields need to be changed then choose Options $\rightarrow$ Edit Author $(\mathrm{Ctrl}+\mathrm{A})$ to display the Author Properties dialog (fig. 5).

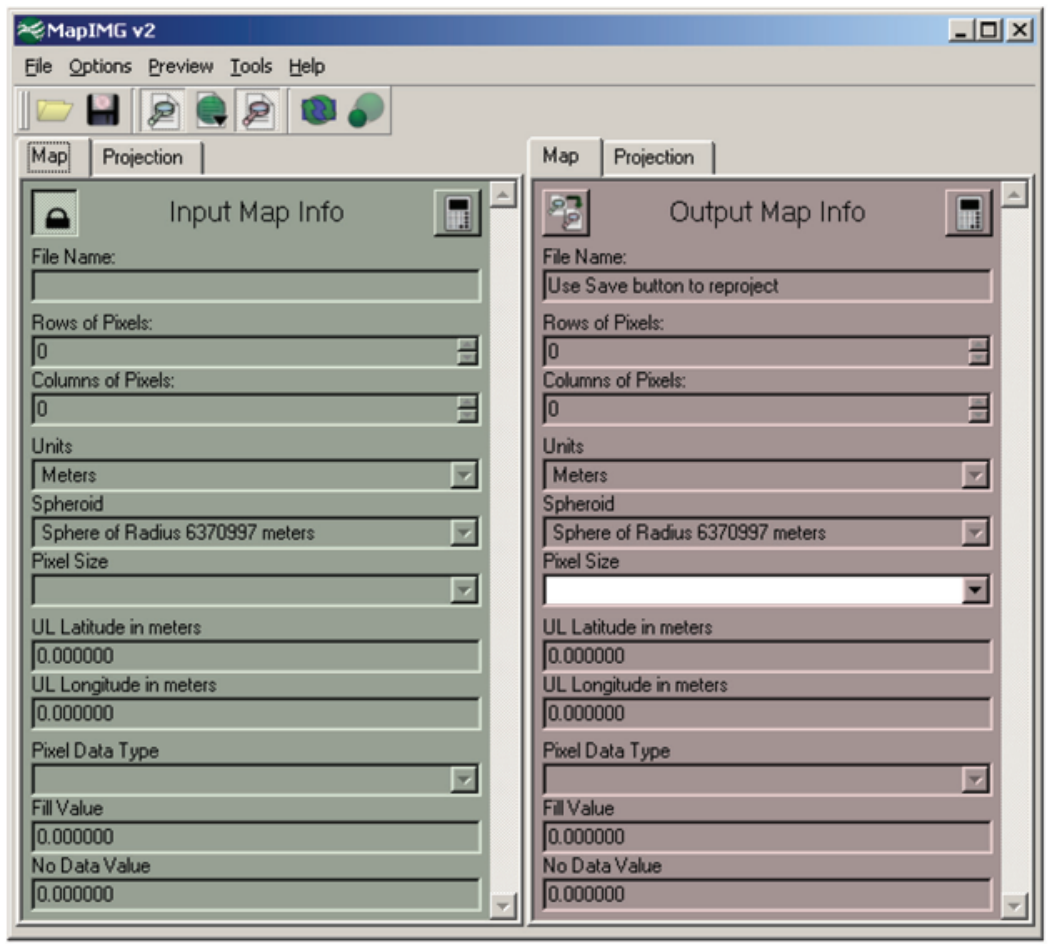

Figure 6. mapIMG Main Dialog. 


\section{Actions Overview}

\section{Toolbar Buttons}

\begin{tabular}{|l|l|}
\hline & $\begin{array}{l}\text { Open: Pressing this button displays the open file dialog and allows the user to } \\
\text { select an IMG or TIFF file and load it as input. } \\
\text { Re-project and Save: Assuming all parameters are filled and error free, press- } \\
\text { ing this button will display the save file dialog and allow the user to select a } \\
\text { destination for their re-projection and specify resampling options. }\end{array}$ \\
\hline $\begin{array}{l}\text { Show Input Parameters: Pressing this button toggles whether the green panel } \\
\text { for input information is shown or not. }\end{array}$ \\
$\begin{array}{l}\text { Show Preview: Pressing this button toggles whether the preview panel is shown } \\
\text { or not. Also, if the user clicks on the down arrow then they can select to show } \\
\text { input or output previews. } \\
\text { Show Output Parameters: Pressing this button toggles whether the red panel } \\
\text { for output information is shown or not. }\end{array}$ \\
$\begin{array}{l}\text { Resample: Pressing this button will resample the viewer to the current resolu- } \\
\text { tion. } \\
\text { (See "Using the Viewer": page 17) } \\
\text { Preview Re-projection : Pressing this button will hide the input parameters and } \\
\text { show the preview panel with a small scale version of what the re-projection will } \\
\text { look like. }\end{array}$
\end{tabular}

\section{Parameter Buttons}

\begin{tabular}{|c|l|}
\hline & $\begin{array}{l}\text { Lock and Unlock: This button is present on the input parameters and allows the } \\
\text { user to unlock and edit them. By relocking the input the parameters are auto- } \\
\text { saved. }\end{array}$ \\
\hline $\begin{array}{l}\text { Calculate: Pressing this button will update the rows, columns, and upper left } \\
\text { coordinates with their values based on the GCTP calculations using all projec- } \\
\text { tion info and the pixel size. }\end{array}$ \\
\hline $\begin{array}{l}\text { Copy from Input: Pressing this button will copy all the values from the input } \\
\text { list to the output list. This is a quick way to make minor adjustments to a projec- } \\
\text { tion. }\end{array}$ \\
\hline
\end{tabular}




\section{Other Actions}

\begin{tabular}{|c|l|}
\hline Eave Input Parameters: This action is redundant to locking the input param- \\
& $\begin{array}{l}\text { Save } \\
\text { eters. It will save the parameters in the list if they are valid and prompt if a file is } \\
\text { about to be overwritten. }\end{array}$ \\
\hline Options & $\begin{array}{l}\text { Edit Author: Choosing this action will reshow the Author Properties dialog } \\
\text { (fig.) so that the user can change their author parameters. }\end{array}$ \\
\hline Tools & $\begin{array}{l}\text { Decision Support System: This action launches the current machine's default } \\
\text { browser to the USGS website hosting the Decision Support System for Map } \\
\text { Projections of Small Scale Data. }\end{array}$ \\
\hline Help & $\begin{array}{l}\text { About mapIMG: Choosing this action will show the About mapIMG dialog } \\
\text { that contains a brief disclaimer, contact info, and a credits section for libraries } \\
\text { used. }\end{array}$ \\
\hline Help & $\begin{array}{l}\text { About Qt: Choosing this action will show the About Qt dialog as per request by } \\
\text { Trolltech, the authors of Qt. }\end{array}$ \\
\hline Meenu & $\begin{array}{l}\text { Exit: This action will immediately exit mapIMG. } \\
\text { Do not pass GO and do not collect \$200. }\end{array}$ \\
\hline
\end{tabular}

\section{Re-Projecting}

The first step in performing a re-projection is to load the desired input file to re-project. This is done by clicking the Open button $(\square)$, choosing File $\rightarrow$ Open..., or pressing $\mathrm{Ctrl}+\mathrm{O}$. An Open File window (fig. 7) will open that is used to select an IMG (generic binary) or TIFF file.

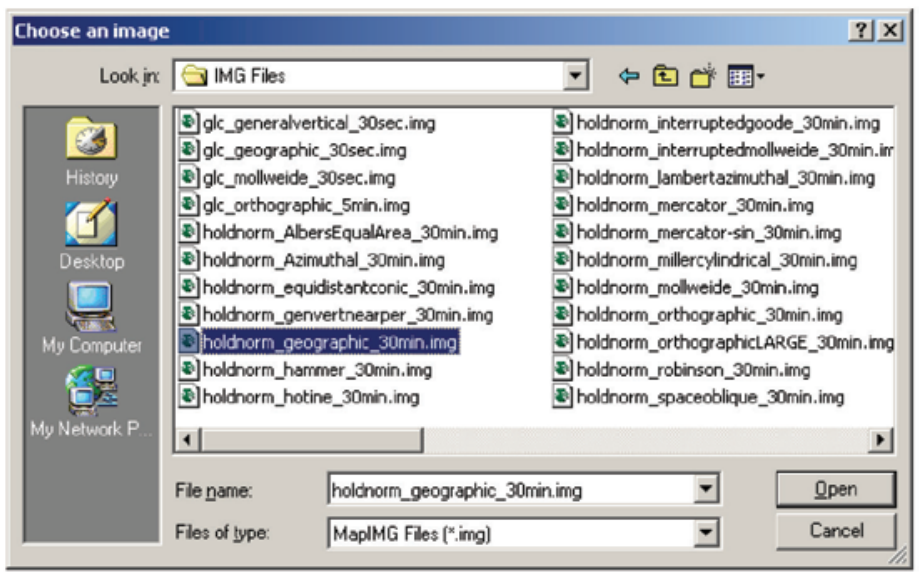

Then click the Open button; if the selected file has an associated XML file, it will be automatically loaded (fig. 8). Otherwise, the input parameters must be entered as outlined in 'Modifying Input Parameters.'

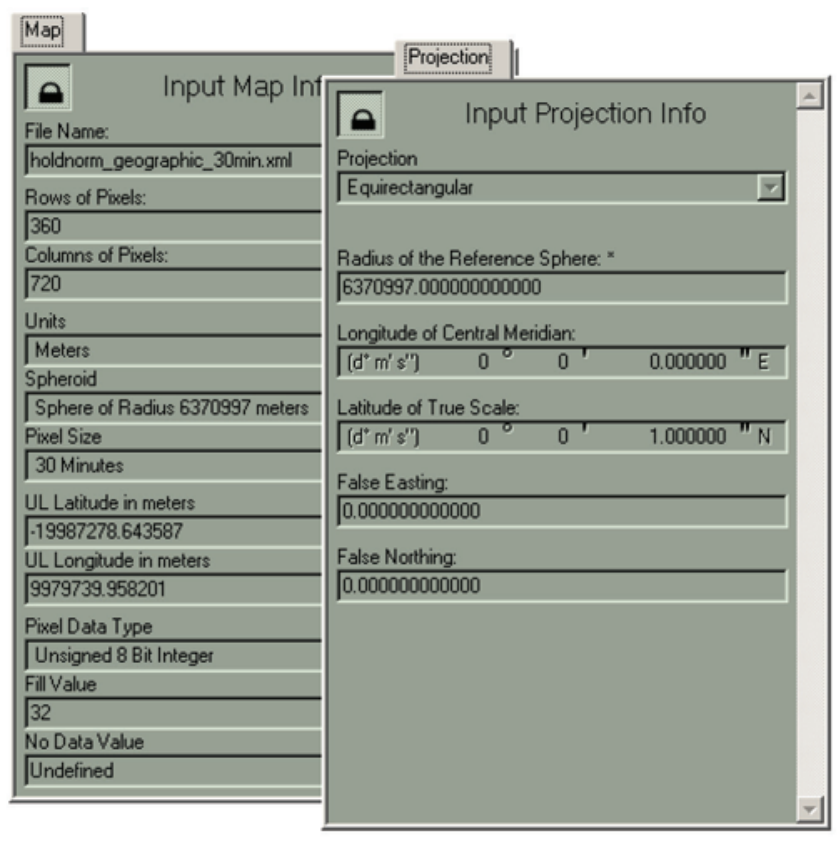

Figure 8. Loaded Input Parameters.

Figure 7. Open File Dialog. 
Next, the output parameters need to be set (fig. 9). Again, this procedure is outlined in 'Modifying Input Parameters.' This process is streamlined by the "Copy from Input" button (를). If the red output parameters are not visible, click the "Show Output Parameters" button (8).

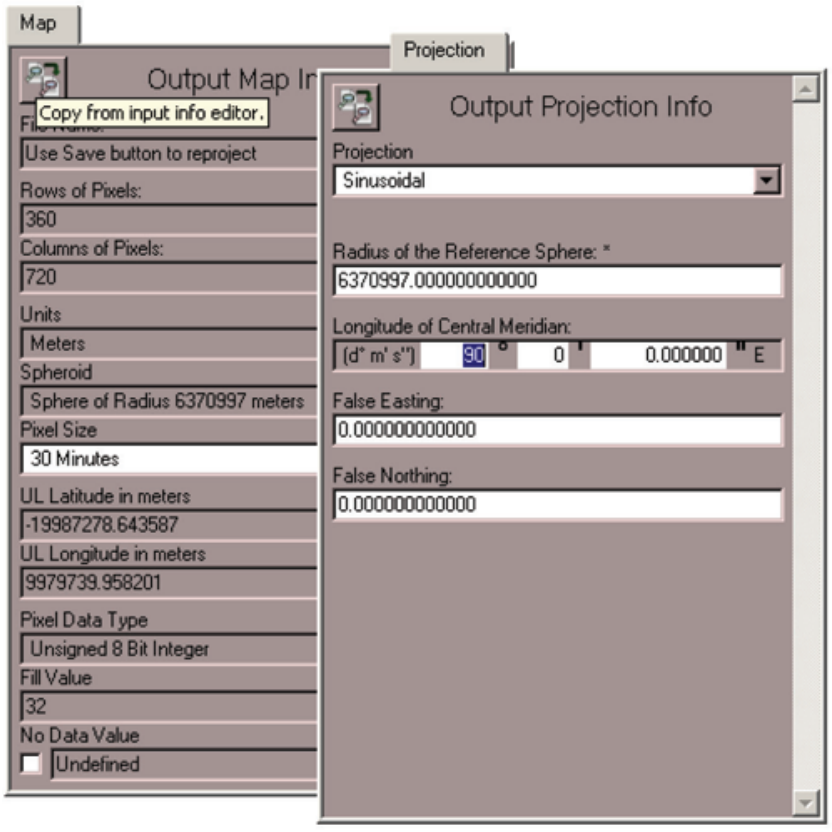

Figure 9. Edited Output Parameters.

Once all the parameters have been set, click the "Reproject and Save" button $(\boldsymbol{\theta})$, choose "File" $\rightarrow$ "Re-project and Save", or press Ctrl+S. Assuming there are no errors or warnings for the parameter choices, the next window shown should be a "Save File" dialog (fig. 10). This window is used to select the destination and name for the new file created after re-projecting. If ".img" is not typed in to the "File Name" field then it automatically will be appended to the file name. Click "Save" to continue.

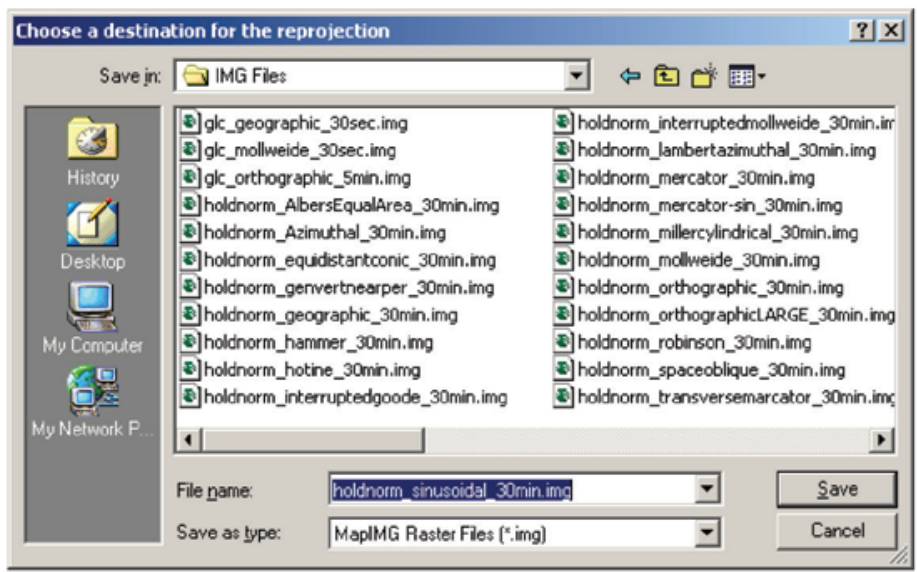

Figure 10. Save File Dialog.
The next window to appear is the "Resample Options" dialog (fig. 11).

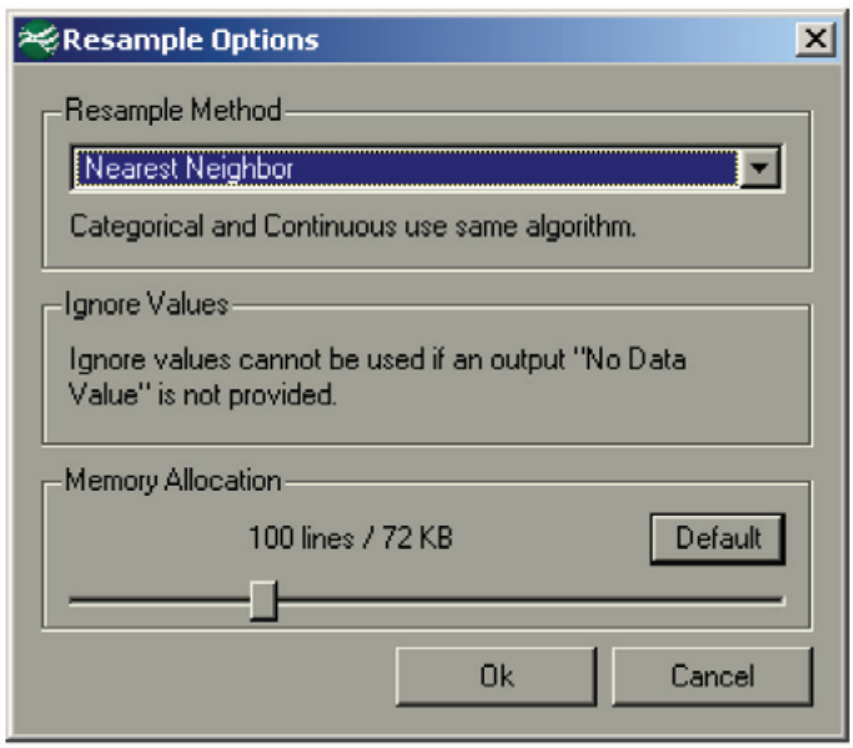

Figure 11. Resample Options Dialog.

After clicking "Ok" the re-projection process begins. A progress bar (fig. 12) is shown, which allows the user to abort the process. The re-projection is complete once the progress bar reaches $100 \%$. If it reaches this point without the user pressing "Abort" then a "Completed" message box will be displayed (fig. 13).

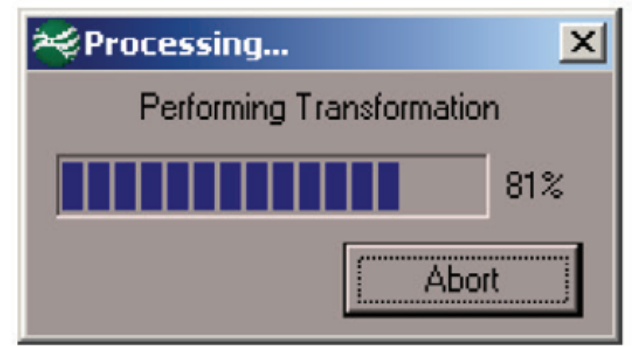

Figure 12. Re-projection Progress.

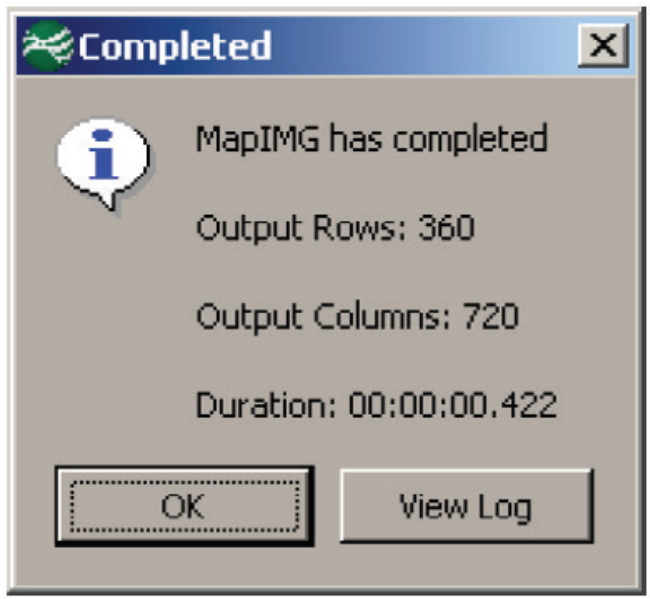

Figure 13. Completed Message Box. 
By clicking "View Log" the user is shown the mapIMG Log dialog (fig. 14), which can be used to save the log of the re-projection to a text file.

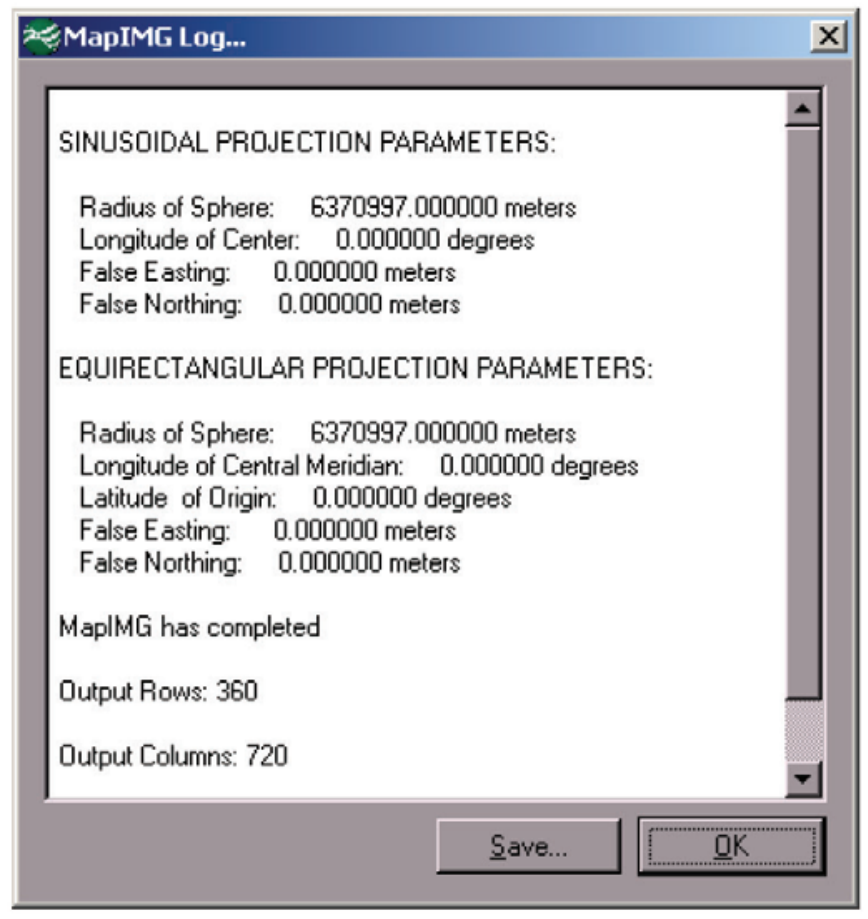

Figure 14. mapIMG Log Dialog.

\section{Modifying Input Parameters}

The first time a file is loaded, or if the paired XML file becomes corrupt beyond recovery the message shown in figure 15 will appear. If a valid XML file was found, the values will be loaded. Press the lock/unlock button $(\boldsymbol{Q}, \boldsymbol{E})$ to change to the unlocked state and then proceed to change parameters as normal.

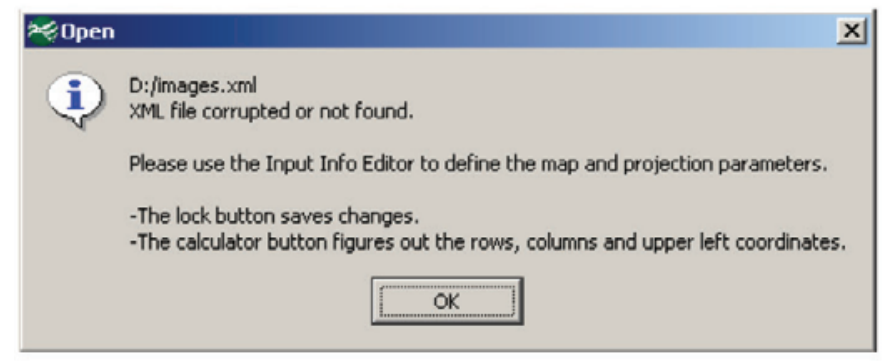

Figure 15. Missing or Corrupt XML.

1. Setup the "Projection" parameters
a. Select the "Projection" tab
b. Select the projection for the input file from the "Projection" drop down menu.

c. The area below the "Projection" drop down menu will change to reflect the parameter relevant to the selected projection. Make all changes required to correspond to the input image. This may include "Radius of the Reference Sphere", "Longitude of Central Meridian", "Latitude of True Scale", "False Easting", "False Northing", or other relevant parameters.

2. Setup the "Map" parameters

a. Select the "Map" tab

b. Select the "Pixel Size" from the "Pixel Size" drop down menu.

c. Manually enter the number of "Rows of Pixels", "Columns of Pixels", "Upper-left Latitude in meters", and "Upper-left Longitude in meters", or press the "Calculate" button ( $)$ to calculate these parameters based on the selected pixel size and projection.

d. Select the pixel data type of the input file from the "Pixel Data Type" drop down menu. An incorrect data type will not only produce incorrect results, but also may cause instabilities in the mapIMG itself.

e. If the input image has a frame around it check the "Fill Value" check box indicating that a "Fill Value" is present. Then, enter the value in the "Fill Value" edit box.

f. If the input image has a value corresponding to areas where data are unavailable, check the "No Data Value" check box. Then, enter this value in the "No Data Value" edit box. An example of this may be a population dataset where areas with an unknown population have a set value of -1 , for instance.

g. To save the XML file corresponding to the input image press the lock/unlock button ( $)$ or choose File $\rightarrow$ Save Input Parameters. If the lock/ unlock button is used the icon should change from unlocked ( $\boldsymbol{Q}$ ) to unlocked $(\boldsymbol{Q})$ and the parameter fields will become read-only and a prompt will ask for confirmation of the input parameters. This indicates a successful save of the XML file.

\section{Modifying Output Parameters}

Once the desired input file has been loaded and its parameters configured the next step is to set up the output projection information. It is suggested that the Copy from Input button ( 
input to the output. Once this is done, only the parameters to be changed will require user interaction. "Pixel Data Type" is set by the input "Pixel Data Type" and cannot be altered.

\section{Setup the "Projection" parameters}

a. Select the "Projection" tab

b. Select the projection that the input file represents from the "Projection" drop down menu. Remember to check for the Bad Projection tag (fig. 1) before continuing. If the tag is present, mapIMG may become unstable or produce invalid output if the output projection is used. A link to the Decision Support System (DSS) for Map Projections of Small Scale Data is provided to assist in selecting an appropriate projection. The link is available under "Tools" $\rightarrow$ "Decision Support System". It will attempt to load the default browser of your operating system to the following address: http://mcmcweb.er.usgs.gov/ DSS/ .

c. The area below the "Projection" drop down menu will change to reflect the parameter relevant to the selected projection. Make all changes required to correspond to the input image. This may include "Radius of the Reference Sphere", "Longitude of Central Meridian", "Latitude of True Scale", "False Easting", "False Northing", or other relevant parameters.

d. Make note of any field with an asterisk (*) next to the parameter label. These parameters have additional constraints that should be taken into account before continuing. Placing the mouse cursor over the label for a few seconds will produce a tool-tip with additional information about the particular constraints of that parameter.

2. Setup the "Map" parameters

a. Select the "Map" tab

b. Select the pixel size from the "Pixel Size" drop down menu.

c. Press the Calculate button ( $\mathbf{\square})$ to calculate "Rows of Pixels", "Columns of Pixels", "Upperleft Latitude in meters", and "Upper-left Longitude in meters" based on the selected pixel size and projection.

Note: Calculated values are for a global extent only.

d. If the input image has a "Fill Value" the output "Fill Value" will be the same, and may not be changed. This prevents having multiple different framing values in the same file. If the input does not have a "Fill Value" it can be manually entered or the "Recommend Fill Value" button (?) can be pressed to generate a new "Fill Value". This is done by taking a small sample of the image and adding two to the maximum value encountered.

e. If the input image has a "No Data Value" the output "No Data Value" will be the same, and may not be changed. This prevents having multiple values indicating where no data are available. If the input does not have a "No Data Value" check the output "No Data Value" check box and manually enter a value in the output "No Data Value" edit box.

Note: A No Data Value is required if Ignore Values are going to be used during the resample process (see 'Choosing Resample Options').

\section{Choosing Resample Options}

After the output file location has been selected using save and re-project, the next step is to choose the resampling options. At any point, the Cancel button may be clicked to end the re-projection process, and the OK button may be clicked to continue on to the re-projection.

1. Choose the resampling method-The available options currently include "Nearest Neighbor", "Sum", "Mean", "Median", "Mode", "Min", and "Max". As the method is selected, text under the choice will change to provide a note on this resampling methods performance in categorical vs. continuous data.

2. Enter all Ignore Values into the list.

3. Adjust memory allocation-Allowing for a larger line cache by increasing the memory allocation can speed up the re-projection and improve accuracy in the sum resampling. This memory is used to store lines of the input image. If a pixel value is needed from the input on a line that was accessed before, it would be much faster to access if it was still located in memory; a large line cache will improve the chances of that happening. The sum resampling method marks pixels in the allocated cache that have been used in the output. If a line is pushed out of the cache and then called upon later, the values may potentially be counted twice. The "Default" value is 100 lines, and the minimum is the ratio of output lines to input lines plus 2 percent of the output lines.

\section{Using the Viewer}

When the viewer is shown with the "Show Preview" button ( it will display a USGS logo by default. Once an input 
file has been loaded the viewer will be forced to input mode and loading of the preview will begin. This process is shown by two progress bars. The first one is creating a color table by sampling the file, and the second one is loading values from the file and drawing them into a picture. Once they are both complete, the preview image will be displayed.

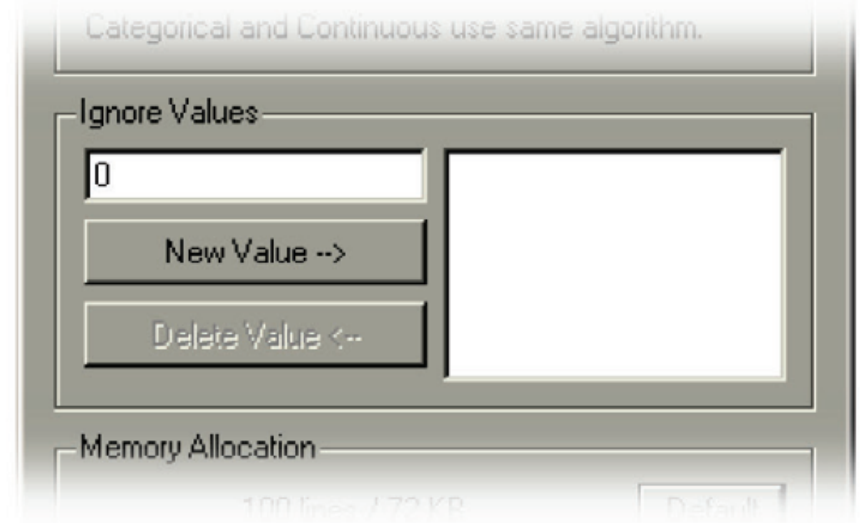

Figure 16. Ignore Values Section.

The preview panel will stretch to fill the mapIMG window. As the window is resized its boundaries will change and the preview picture within it will be stretched to maintain the original aspect ratio and moved to stay centered in the panel. The image previewed is a down-sampled image using nearest neighbor. Therefore, when the image is stretched, the individual pixels become larger without resampling the file. Once a new size for the view is determined, the "Resample" button (B) can be clicked and mapIMG will resample the file at the current resolution to produce a higher quality image.

Note that the "Show Preview" button has a down arrow on the lower right corner. Clicking and holding on this arrow will show a pop-up menu allowing the user to switch between "Preview Input" and "Preview Output". Choosing preview output is similar to pressing the "Preview Re-projection " button (-). The preview panel will be displayed and four progress bars will complete in the process. The first one represents the down-sampling of the original image to a much smaller size. The second one is a fast re-projection to a low resolution version of the output projection parameters. The final two progress bars are the color table generation and value loading as previously outlined.

\section{Other Tools}

Currently (2012), the only extra tool with mapIMG is the Decision Support System for Map Projections of Small Scale Data (DSS). A link is available under "Tools" $\rightarrow$ "Decision Support System". It will attempt to load the default browser of your operating system to the following address: $h t t p: / /$ mcmcweb.er.usgs.gov/DSS/. A user's guide is available for the DSS and is available at http://carto-research.er.usgs.gov/ projection/pdf/DSS_User_Guide.pdf.

\section{Troubleshooting}

Error, missing or malformed $<\ldots \quad>$ tag under $<\ldots>>$. $X M L$ File corrupt or not found.-These errors occur when there is an issue with the $\mathrm{xml}$ metadata file. The solution is to use the input parameter editor and save changes by using the lock button ( $\mathbf{P})$.

Tiff Image - Conversion failed for unknown reason.-The Tiff library couldn't open the selected Tiff file. Verify that the file has a tif extension and is truly a Tiff image file.

mapIMG cannot read this file.-Since mapIMG works with Generic Binary files, the only way of discriminating file types is by checking the file's extension. Make sure the file ends in .img. A common error is to use an ERDAS Imagine or Idrisi file, both of which also use the same extension of .img.

Save Input Parameters - File already exists. Do you want to replace it? - This is a safeguard to protect the metadata file from being replaced if an accidental change is made while the file is unlocked. Click "yes" to replace the file with the new parameters or "no" to undo all changes and revert the parameters back to how they were last saved.

mapIMG-Unable to launch web browser to...-This error occurs when trying to access the DSS using the Tools menu in mapIMG. The solution is to copy and paste the URL to your preferred browser. http://mcmcweb.er.usgs.gov/ DSS/

The current spheroid code and/or unit code are unsupported.-The spheroid and unit code somehow became modified. This should not be possible with the current user interface.

Pixel Size must be greater than 0.-Make sure the Pixel Size parameter for the input and output are blank or zero.

Rows and Columns must be greater than 0.-These values usually can be generated by the calculator button ( $\mathbf{\square})$. If that doesn't work, then you are in either an unsupported "bad projection," or one of the parameters is set in such a way that the final resolution would be very close to zero (that is, True Scale of Transverse Mercator shouldn't be zero).

Please select a valid data type for each pixel.Select a data type using the drop down box in the input parameters.

If any other errors or unexpected results occur please contact the authors of the mapIMG software package by email at mapIMG@usgs.gov.

\section{References Cited}

Elassal, A.A., 1987, General Cartographic Transformation Package (gctp), Version II: NOAA Technical Report no. 124 cgs $9,24 \mathrm{p}$.

ERDAS Inc., 1999, ERDAS field guide (5th ed.): Atlanta, Ga. 
Finn, M.P., and Trent, J.R., 2004, Users guide for the mapIMG re-projection software package, version 1.01: U.S. Geological Survey Open-File Report 2004-1394, 11 p.

Slater, P.N., 1980, Remote sensing-Optics and optical systems: Reading, Mass., Addison-Wesley Publishing Company, Inc., 593 p.

Usery, E.L., Finn, M.P., Cox, J.D., Beard, T., Ruhl, S., and Bearden, M., 2003a, Projecting global datasets to achieve equal areas: Washington, D.C., ACSM, Cartography and Geographic Information Science, v. 30, no. 1, p. 69-79.
Usery, E.L., Seong, J.C., Steinwand, D., and Finn, M.P., 2003b, Accurate projection of small-scale raster datasets in ICA 2003: Proceedings of the 21st International Cartographic Conference: Cartographic Renaissance, Durban, South Africa, 10-16 August, International Cartographic Association, p. 1,174-1,180.

Usery, E.L., Seong, J.C., Steinwand, D., and Finn, M.P., 2001, Methods to achieve accurate projection of regional and global raster databases: U.S. Geological Survey Open-File Report 01-383, $11 \mathrm{p}$. 
Publishing support provided by:

Rolla Publishing Service Center

For more information concerning this publication, contact:

Director, USGS Center of Excellence for Geospatial Information Science (CEGIS)

1400 Independence Road

Rolla, M0 65401

(573) 308-3837

Or visit the CEGIS Web site at: http://cegis.usgs.gov 


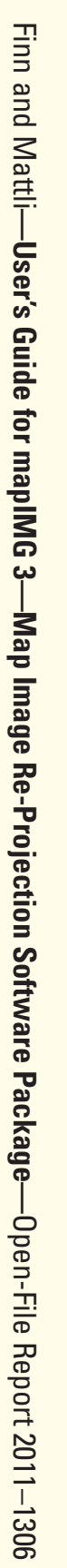

